\title{
Demographic Factors That Influence United States Medical Licensing Examination Scores
}

\author{
Shafaq Jawed, MBBS \\ Jinnah Sindh Medical University, Karachi, Pakistan
}

\section{TO THE EDITOR}

The United States Medical Licensing Examination (USMLE) is a standardized 3-step examination required for obtaining medical licensure in the United States. Medical students who are planning to attempt the USMLE must be aware of the demographic factors that influence a student's score.

Increasing age is generally associated with greater levels of intellect and improved critical thinking because of the increase in practical life experiences. However, the literature reveals a variable association of age with USMLE scores. Gauer and Jackson found that traditional age students (defined as $<25$ years old at matriculation) scored higher on USMLE Step 1 and Step 2 compared to nontraditional age students. ${ }^{1}$ On the other hand, in a study of 2 consecutive second-year medical student classes, Giordano et al showed that USMLE Step 1 scores did not correlate with either age or sex. ${ }^{2}$ Ogunyemi and Taylor-Harris compared USMLE Step 2 scores with demographic factors of 171 students and showed a negative correlation between age and examination scores $(r=-0.405, P=0.000)$ but a positive correlation between Step 1 scores and Step 2 scores $(r=0.681, P=0.000){ }^{3}$

Financial stability, methodology for learning and retaining knowledge, and clinical teaching tools have stronger associations with USMLE scores than a student's age..$^{2-5}$ A survey of USMLE Step 1 scores and demographic information for second-year medical students who took the examination revealed that students who did not receive financial need-based scholarships scored higher on the USMLE Step 1 compared to students who received such scholarships (mean scores of 243.0 vs 234.9 , respectively, $P=0.01)^{2}$

Learning approach has a significant impact on a student's ability to understand and retain knowledge. In a yearlong study at The Ohio State University, senior students who had recently completed the Step 1 examination designed and taught a weekly course for second-year medical students. Participants in the course scored higher on the Step 1 examination than nonparticipants (mean scores of 231.25 vs 223.2, respectively, $P=0.005){ }^{4}$

As the USMLE is designed to assess a student's understanding of the basic medical sciences and integration in the clinical setup, third-year medical students who took a course designed to teach the basic science underpinnings of disease scored higher on the USMLE Step 1 and Step 2 examinations compared to medical students in prior years who did not take the class. ${ }^{5}$

Medical students - whether trained in the United States or abroad-must acknowledge the importance of the factors that directly or indirectly influence USMLE scores and adopt strategies and techniques that will help them attain scores on the US standardized examinations that will help qualify them for US residencies.

\section{REFERENCES}

1. Gauer JL, Jackson JB. Relationships of demographic variables to USMLE physician licensing exam scores: a statistical analysis on five years of medical student data. Adv Med Educ Pract. 2018 Jan 10;9:39-44. doi: 10.2147/AMEP.S152684.

2. Giordano C, Hutchinson D, Peppler R. A predictive model for USMLE step 1 scores. Cureus. 2016 Sep 7;8(9):e769.

3. Ogunyemi D, Taylor-Harris D. Factors that correlate with the U.S. Medical Licensure Examination Step-2 scores in a diverse medical student population. J Natl Med Assoc. 2005 Sep;97(9):1258-1262.

4. Alcamo AM, Davids AR, Way DP, Lynn DJ, Vandre DD. The impact of a peer-designed and -led USMLE Step 1 review course: improvement in preparation and scores. Acad Med. 2010 Oct;85(10 Suppl):S45-S48. doi: 10.1097/ACM.0b013e3181ed1cb9.

5. Brownfield EL, Blue AV, Powell CK, Geesey ME, Moran WP. Impact of the foundations of clinical medicine course on USMLE scores. J Gen Intern Med. 2008 Jul;23(7):1002-1005. doi: 10.1007/s11606-008-0631-z. 